\title{
Epizootie de pasteurellose dans un élevage semi-intensif de lapins de race locale au Sénégal
}

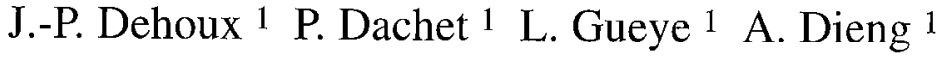

A. Buldgen 2

\section{Mots-clés}

Lapin - Pasteurellose - Pasteurella multocida - Klebsiella pneumoniae Pseudomonas acrogenes - Saison humide - Epizootie - Vaccination Sénégal.

\begin{abstract}
Résumé
Une épizootie de pasteurellose s'est déclenchée dans un élevage semi-intensif de lapins (200 animaux) de race locale, à Thiès (ENSA) au Sénégal, pendant la saison des pluies 1995 (août à octobre). L'épizootie a provoqué la mort de 87 animaux. Les jeunes ont été particulièrement touchés par l'affection. Des symptômes tels que jetage, conjonctivite, perte de l'œil, otite moyenne et interne étaient observés chez les lapereaux tandis que les adultes manifestaient de la parésie postérieure. Pasteurella multocida, Klebsiella pneumoniae et Pseudomonas aerogenes ont été identifiés. Un antibiogramme a révélé une sensibilité de ces germes au chloramphénicol, à l'association sulfaméthoxazole/trimćthoprime et à la colistine. Les températures et l'humidité élevées caractérisant la saison des pluies semblent avoir favorisé l'apparition de la maladie à partir de porteurs sains issus du noyau fondateur de l'élevage. Un traitement à la colistine et au chloramphénicol a été instauré avant une vaccination de tous les animaux contre la pasteurellose.
\end{abstract}

\section{INTRODUCTION}

Le lapin est une source intéressante de protéines en zone tropicale, en particulier pour les populations rurales et périurbaines disposant de faibles ressources monétaires. Son élevage est favorisé par le faible coût d'achat des animaux, par la multiplication rapide de ses effectifs et par des besoins nutritifs relativement aisćs à satisfaire (7). Les propriétés de cet élevage ont provoqué un intérêt croissant pour la cuniculiculture au Sénégal. Les effectifs de base excèdent toutefois rarement cinq femelles reproductrices. Par ailleurs, de nombreuses maladies, encore peu connues, sévissent dans les clapiers et sont en grande partie responsables de la faible productivité des élevages (4).

Dans le cadre du Projet d'appui au Département des productions animales de l'ENSA (Thiès, Sénégal), un volet d'études consacré au lapin local a été créé afin d'approfondir la connaissance de sun élevage et de sa productivité. Cette note reprend les observations faites lors d'une épizootie de pasteurellose qui s'était déclenchée dans la lapinière de l'ENSA pendant la saison pluvieuse 1995.

\section{SITUATION EPIDEMIOLOGIQUE}

Les lapins de race locale étaient issus d'un noyau reproducteur acheté sur les marchés environnant Thiès en octobre 1994. D'un poids moyen adulte de $2,5 \mathrm{~kg}$, les sujets étaient élevés dans un bâ-

1. Département des productions animales, Ecole nationale supérieure d'agriculture (ENSA), BP A 296 (RP), Thiès, Sénégal

2. Unité de zootechnie, Faculté universitaire des sciences agronomiques, Passage des Déportés 2, 5030 Gembloux, Belgique timent en béton semi-ouvert occupant une superficie de $30 \mathrm{~m}^{2}$. Ils étaient logés individuellement (reproducteurs) ou par lot (engraissement) dans des cages métalliques disposées en batterie sur 2 ou 3 étages. En août 1995, l'effectif total de la lapinière atteignait 193 sujets. Ces derniers étaient nourris au moyen de fourrages verts (Leucaena leucocephala, Andropogon gayanus) complémentés par un aliment granulé du commerce. Ils disposaient en permanence d'eau claire.

Aucune prophylaxie particulière n'a été instaurée, mis à part une administration bimensuelle d'anticoccidien dans l'eau de boisson (sulfadimidine/diavéridine). La lapinière était nettoyée tous les jours : les matières fécales étaient enlevées et la dalle rincée au Crésyl. Les cages étaient fréquemment lavées à l'eau de Javel.

La maladie est apparue durant le mois d'août 1995 et a sévi jusqu'en octobre de la même année. Cette période a coïncidé avcc la saison des pluies qui s'étend de juin à octobre avec les précipitations maximales en août et septembre. A cette époque de l'année, la température moyenne journalière minimale dans la lapinière était de $25,6^{\circ} \mathrm{C}$. La température moyenne maximale atteignait $35,5^{\circ} \mathrm{C}$ et l'humidité relative moyenne de l'air ambiant avoisinait 70 p. 100.

\section{SYMPTOMATOLOGIE}

D'août à octobre 1995,87 mortalités imputables à la maladie ont été recensées. Au total, 5 adultes, 45 lapereaux sevrés et 37 lapereaux sous la mère sont morts ou ont été sacrifiés (animaux agonisants ou incurables). Les jeunes animaux ont été le plus frappés par la maladie (tableau I). Alors que le taux de mortalité moyen dans l'élevage est de 4,5 p. 100, durant les trois mois de l'épizon- 
TABLEAU I

\section{Evolution des mortalités suivant la catégorie d'animaux el le mois de l'année}

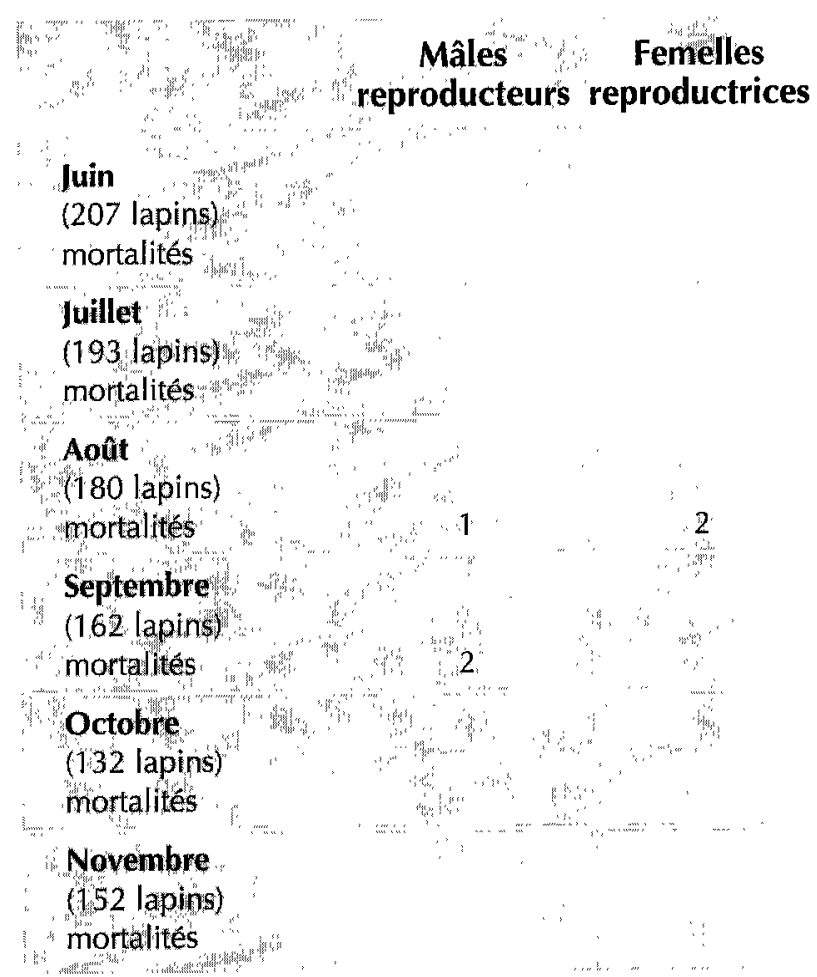

tie, ce taux était respectivement de 16,6 p. $100,21,6$ p. 100 et 16,6 p. 100. Le taux de létalité était également très élevé atteignant respectivement 84,5 p. $100,91,7$ p. 100 et 82,3 p. 100.

La symptomatologie est apparue très différente suivant la catégorie d'animaux. Les jeunes animaux, sevrés ou sous la mère, présentaient des manifestations cliniques spécifiques. Un léger jetage séreux puis muqueux était constaté avec de rares manifestations de toux ou d'éternuements. Les poils des pattes antérieures étaient collés et recouverts par un mucus jaunâtre séché que le lapin essuyait en se frottant le nez. Un à deux jours plus tard, alors que l'animal manifestait des signes d'abattement et d'anorexie, une conjonctivite unilatérale est apparuc dans la majorité des cas avec dépôt d'un film muqueux blanchâtre sur la cornée de l'œil atteint. I a conjonctivite, d'abord séreuse, devint ensuite purulente. Un ankyloblépharon purulent soudait les paupières. La cornée était perforée avec une présence abondante de pus dans les chambres entraînant par la suite la perte de l'œil. L'animal présentait ensuite une atteinte de l'oreille moyenne et interne. Ceci se manifestait par une posture anormalc avec rotation du cou et de la tête du côté de l'œil atteint. L'animal mourait un à deux jours plus tard. Certains jeunes lapereaux développaient aussi des signes nerveux : parésie postérieure avec incontinence urinaire et fécale. Les adultes ne présentaient que la symptomatologie nerveuse de type méningoencéphalitique : parésie postérieure, paralysie et incontinence. Chez les survivants, les paramètres de fécondité étaient toutefois en baisse : les femelles avaient des portées de taille réduite (3 lapereaux contre 5 en moyenne pour l'élevage) avec des cas d'hydrocéphalie. Le nombre de services pour avoir une gestation était passé de un à trois.

A l'autopsie, mis à part les lésions spécifiques au niveau de l'œil et de l'oreille moyenne, les lésions anatomo-pathologiques étaient peu caractéristiques : décoloration du foie et des reins, lésions d'entérite chez certains animaux. L'ouverture de la boîte crânienne ne révéla aucune lésion macroscopique dans les cas de parésie postérieure.

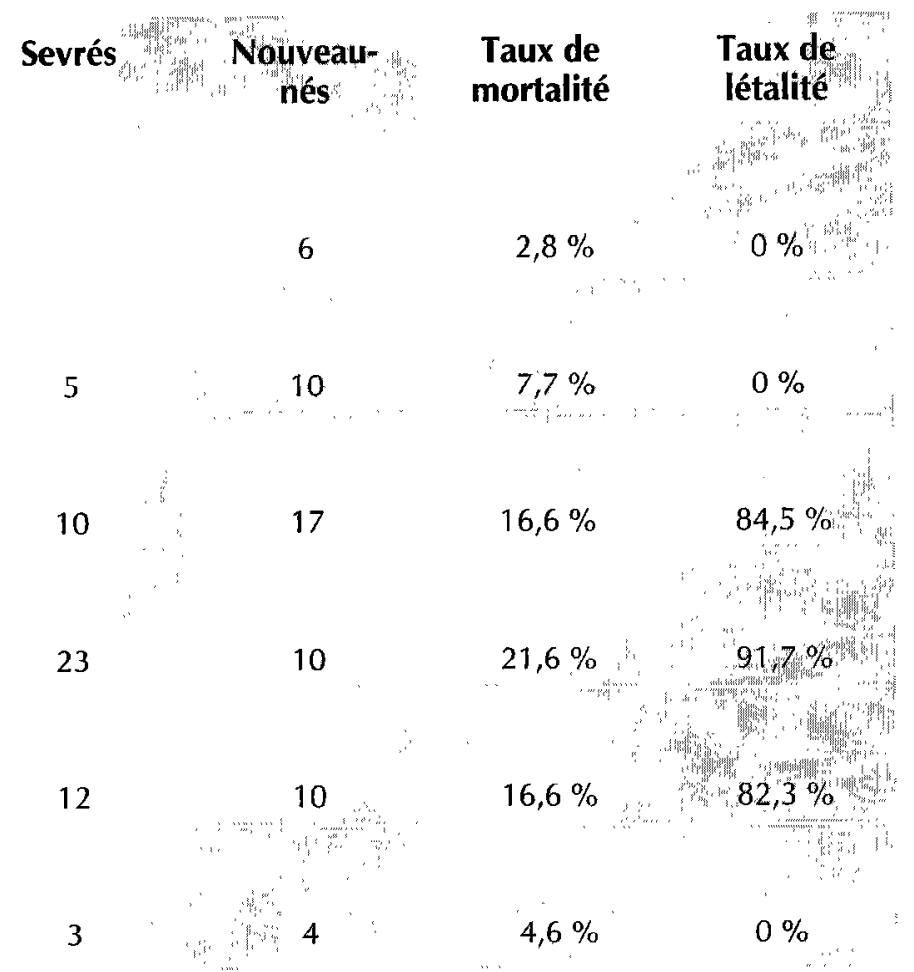

L'administration d'oxytétracycline par voics parentérale ct orale et d'un onguent ophtalmique (chlortétracycline) à tous les animaux durant le mois d'août a permis de constater une rémission dés symptômes. Néanmoins, une nouvelle flambée de la maladie était constatée en septembre.

\section{IDENTIFICATION DE L'AGENT RESPONSABLE}

En octobre 1995, des lapins malades ont été envoyés dans les départements de diagnostics virologiques et bactériologiques de l'hôpital Saint-Jean-de-Dieu, à Thiès et au laboratoire de diagnostic vétérinaire de Haan, à Dakar. Pasteurella multocida, Klebsiella pneumoniae et Pseudomonas aerogenes ont été isolés. Un antibiogrammc a révélé une sensibilité de ces germes à la colistine, au chloramphénicol et à l'association sulfaméthoxazole/triméthoprime. Listeria monocytogenes, Bordetella bronchiseptica et Thelazia spp. n'ont pas été identifiés malgré une demande diagnostique précise.

Lorsque le diagnostic a été prononcé, un traitement à base de chloramphénicol par voie parentérale $(100 \mathrm{mg} / \mathrm{kg})$ et conjonctivale a été instauré à la fin du mois d'octobre. Une première vaccination contre la pasteurellose (Pasteurellad R - Laboratoire national de l'Elevage et de Recherches vétérinaires-Dakar) a ćté réalisée dix jours plus tard avec un rappel trois mois après.

\section{DISCUSSION}

La pasteurellose se manifeste chez le lapin sous de nombreuses formes cliniques : thinite (coryza), pneumonie, otite, abcès, mammite, pyomètre, orchite et septicémie. Okerman et coll. (6) ont montré que les souches de Pasteurella isolées chez le lapin se différencient fortement d'après leur virulence. Les souches isolées dans les cas de rhinite sont nettement moins virulentes que celles 
isolées dans les formes de pneumonie et de septicémie. Dans cette étude, la symptomatologie respiratoire s'est avérée peu importante lors d'observations cliniques. La perte de l'œil ainsi que les manifestations cliniques de l'atteinte de l'oreille sont autrement plus fréquentes. Ces manifestations, ainsi que celles de type méningoencéphalitique chez l'adulte, sont à mettre en parallèle avec les symptômes décrits par IIouchot (2). Cet auteur observait des signes cliniques semblables dans ses clapiers, en émettant l'hypothèse d'une pasteurellose. Klebsiella pneumoniae avait été également isolé dans les prélèvements.

Parfois jusqu'à 90 p. 100 des animaux de certains élevages (5) sont des porteurs sains. Ces sujets excrètent la bactérie dans le milieu ambiant lors de conditions de stress, telles que des températures élevées associées à des taux d'humidité importants en saison des pluies. Depuis l'achat diı noyau de départ en octobre 1994 , aucun autre animal n'a été introduit dans l'élevage. Il est donc vraisemblable qu'un ou plusieurs des animaux étudiés aient été des porteurs sains. D’après Lukefahr et Cheeke (4), pour éviter ce problème de portage, certains projets ont été réalisés en démarrant des élevages de lapins à partir de lignées SPF (specific pathogenfree) créées et développées dans certaines universités et laboratoires. Toutefois ce genre de lignéc n'cxiste pas au Sénégal.

Bien que l'immunité conférée après guérison soit faible (6), les adultes ont peu souffert de la maladie alors que les lapereaux connaissaient des taux de morbidité et de mortalité élevés.

Le traitement de cette affection est décevant et difficile. Les soins procurent souvent une rémission temporaire des symptômes. Selon les auteurs, des antibiotiques comme le chloramphénicol, l'oxytétracycline, la streptomycine sont recommandés $(8,9)$. D'après Spanogne et Okerman (10), sans mesures sanitaires importantes (lavage, désinfection, conduite d'élevage rationnelle, contrôle des conditions d'ambiance et élimination des porteurs), il ne faut pas s'attendre à des résultats spectaculaires au moyen de vaccins inactivés. Dans les exploitations importantes, la préparation de vaccins homologues donne de meilleurs résultats que les vaccins polyvalents vendus dans le commerce, surtout lorsqu'ils sont administrés par voie intradermique (1). La vaccination des lapins est préconisée à 4 semaines d'âge, avec rappel vaccinal tous les 3 à 6 mois (1, $8,10)$. Le Normand $(3)$ conseille en outre une prophylaxie médicamenteuse (oxytétracycline, spiramycine, sulfamides) dans l'eau de boisson ou dans les aliments selon une fréquence de 10 jours tous les 2 mois.

\section{- CONCLUSION}

Avec la coccidiose, la pasteurellose est une des maladies les plus redoutables des clapiers. Cette pathologie, très meurtrière chez les jeunes animaux, présente des manifestations cliniques très variées. L'épizootie se déclenche à la suite de conditions de stress à partir d'animaux porteurs sains difficiles à détecter dans les conditions locales. Etant donné que le traitement de cette affection est difficile et décevant, la vaccination des animaux constitue une mesure de prophylaxie indispensable qu'il faut associer impérativement à des conditions sanitaires rigoureuses. L'utilisation d'un autovaccin sera chaque fois préconisée dans les élevages importants car les vaccins vendus dans le commerce sont nettement moins efficaces.

\section{BIBLIOGRAPHIE}

1. HERVOUET P., DONNION C., THIBAULT E., RETAILLEAU B., 1992. Du nouveau dans les autovaccins "maladies respiratoires" : I'utilisation de la voie intradermique. Cuniculture, 107 (19/5) : 215-218.

2. HOUCHOT A., 1981. Bilan pathologique de quatre années d'élevage du lapin domestique servant à la nourriture de glossines (Giossina paloalis gambiensis Vanderplank, 1949) en Haute-Volta. Revue Elev. Méd. vét. Pays trop., 34 (4) : 369-376.

3. LE NORMAND B., 1994. Mesures de prophylaxie indispensable en élevage cunicole : intérêt et coûts. Cuniculture, 119 (21/5) : 229-232.

4. LUKEFAHR S.D., CHEEKE P.R., 1990. Rabbit project planning strategies for developing countries. II : Research applications. Livest. Res. rural Dcv., 2 (3) : 34-47.

5. MERCK, SHARP, DOHME, 1986. Management, husbandry, and diseases of rabbits, In: Fraser C.M. en., The Merck veterinary Manual, 6th ed. Rahway, USA, Merck \& Co. Inc., p. 992-993.

6. OKERMAN L., SPANOGNE L., DEBRUYCKER R.M., 1979. Experimental infection of mice with Pasteurella multocida strains isolated from rabbits. J. comp. Path., $89: 51-55$.

7. OWEN J.E., 1976. Rabbit production in tropical developing countries: a review. Trop. Sci., 18 (4): 203-210.

8. SABATIER H., 1971. Le lapin et son ćlcvage professionnel. Paris, France, Dunod, p. 120-122. (Problèmes de l'entreprise agricole $n^{\circ} 7$ )

9. SCHALL H., 1992. Le lapin. In : Gabrisch K., Zwart P. conrd., La consultation des nouveaux animaux de compagnie. Maisons-Alfort, France, Editions du Point vétérinaire, p. 6-7.

10. SPANOGNE L., OKERMAN L., 1987. Prévention de la pasteurellose des lapins par vaccination au moyen d'un vaccin inactivé : observations sur le terrain. Revue Agric, 5 (40) : 1283-1293.

Reçu le 19.1.96, accepté le 2.8.96 


\section{Summary}

Dehoux J.-P., Dachet P., Gueye L., Dieng A., Buldgen A. Epizootic pasteurellosis in a semi-intensive breeding farm of indigenous Senegalese rabbits

Epizootic pasteurcllosis appeared in a semi-intensive breeding farm (200 animals) of indigenous rabbits in Thiès (ENSA), Senegal, during the 1995 wet season (August to October). It provoked death in 87 animals. Young animals were particularly sensitive to the disease. Nasal discharge, conjonctivitis, eye loss and otitis media and interna were observed in young rabbits while posterior paresis was noted in mature rabbits. Pasteurella multocida, Klebsiella pneumoniae and Pseudomonas aerogenes were identified. An antibiogram revealcd the germs were sensitive to chloramphenicol, sulfamethoxazole/trimethoprim and colistin. High temperatures and humidity during the wet season may have contributed to the outbreak of the disease from healthy carriers introduced at the founding of the farm. Colistin and chloramphenicol treatments were administered before vaccinating all rabbits against pasteurellosis.

Key words: Rabbit - Pasteurellosis - Pasteurella multocida Klebsiella pneumoniae - Pseudomonas aerogenes - Wet season - Epizootics - Vaccination - Senegal.

\section{Resumen}

Dehoux J.-P., Dachet P., Gueye L., Dieng A., Buldgen A. Epizoótia de pasteurelosis en un criadero semi intensivo de conejos de la raza local de Senegal

Se declaró una epizoótia de pasteurelosis en una criadero semi intensivo de conejos (200 animales) de raza local, en Thiés (ENSA), en Senegal, durante la estación de las lluvias en 1995 (de agosto a octubre). La epizoótia provocó la muerte de 87 animales. Los jóvenes fueron particularmente afectados por la infección. En los gazapos se observaron los siguientes síntomas: descarga nasal, conjuntivitis, pérdida de la visión, otitis media e interna, mientras que los adultos manifestaron paresia posterior. Se identificaron Pasteurella multocida, Klebsiella pneumoniae y Pseudomonas aerogenes. El antibiograma reveló la sensibilidad de estos gérmenes al cloranfenicol, a la asociación sulfametoxasol/trimetroprin y a la colistina. Las altas temperaturas y la humedad características de la estación lluviosa, parecen haber favorecido la aparición de la enfermedad a partir de portadores sanos, dentro del núcleo original del criadero. Un tratamiento con colistina y cloranfenicol se instauró, antes de la vacunación de todos los animales contra la pasteurelosis.

Palabras clave : Conejo - Pasteurelosis - Pasteurella multocida - Klebsiella pneumoniae - Pseudomonas aerogenes - Estación humeda - Epizoótia - Vacunación - Senegal. 